\title{
Stabilization for the T-S fuzzy system with the constrained control
}

\author{
Chung-Hsun Sun ${ }^{1}$ and Wen-June Wang ${ }^{2} *$ \\ ${ }^{1}$ Department of Mechanical and Electro-mechanical Engineering, Tamkang University, Tamsui, Taiwan \\ ${ }^{2}$ Department of Electrical Engineering, National Central University, Jhongli, Taiwan \\ *Email: wjwang@ee.ncu.edu.tw
}

\begin{abstract}
In this study, the stabilization for the Takagi-Sugeno (T-S) fuzzy discrete system with constrained controls is investigated. The derived stabilization conditions are based on the Lyapunov stability criterion with the piecewise quadratic Lyapunov function. The constrained control inputs are used to assess the possible state transition, following which the stabilization conditions are reduced. The corresponding Lyapunov inequalities of the interactions among the state transition are integrated into a single matrix to relax the stabilization conditions. Finally, the effectiveness of the proposed stabilization conditions is demonstrated by a numerical example.
\end{abstract}

Keywords: Takagi-Sugeno fuzzy system, constrained control, piecewise Lyapunov function.

\section{Introduction}

During the past decades, the Takagi-Sugeno (T-S) fuzzy control system has been widely explored (see [1$2]$ and references therein). A nonlinear system can be converted into a T-S fuzzy model through the local approximation method or sector nonlinearity method [1]. Subsequently, the T-S fuzzy model-based control design methods can apply to the nonlinear system. The parallel distributed compensation (PDC) [3] control law combined with the Lyapunov stability criterion is usually synthesized to the T-S fuzzy model. The stabilization conditions are usually represented in the linear matrix inequality (LMI) form. Then, the T-S fuzzy modelbased control is completed by using effective LMI tools to solve the LMI problem. The T-S fuzzy model-based control is also successive applied to the system with constrained control. In [4], the control input constraint is represented as the LMIs and solved with the stabilization conditions simultaneously. The LMIs of the control input constraint assure that the designed control satisfies the constraints in the control inputs and have been successively applied to many applications [1-8]. However, the stabilization conditions and input constraint LMIs of the most studies are derived based on the common quadratic Lyapunov function.

The primary Lyapunov stabilizations of the T-S fuzzy control systems are based on the common quadratic Lyapunov function [1-7]. Based on the common quadratic Lyapunov function, the stabilization proce- dure is to obtain a common positive definite matrix such that all the Lyapunov inequalities are satisfied. The Lyapunov inequalities are with respect to the fuzzy rules of the T-S fuzzy system. The common quadratic Lyapunov function based control design is simple but the structure information of the fuzzy rules is unnoticed in the stabilization conditions. In contrast with the common quadratic Lyapunov inequalities, the piecewise quadratic Lyapunov function involves the structural information of the fuzzy rules $[2,8]$. In addition, the piecewise quadratic Lyapunov function has more Lyapunov function candidates than the common quadratic Lyapunov function. Hence, the piecewise quadratic Lyapunov function derives more relaxed stabilization conditions than the common quadratic Lyapunov function does.

The aim of this study is to stabilize the T-S fuzzy discrete system with the constrained control. First, the $\mathrm{T}-\mathrm{S}$ fuzzy system is converted into an equivalent switching T-S fuzzy discrete system. Then, the stabilization conditions are derived based on the Lyapunov stability criterion with the piecewise quadratic Lyapunov function. The constrained control inputs are employed to estimate the possible state transition in advance. Subsequently, only the Lyapunov inequalities associated with the possible state transition are necessary to fulfill. In addition, the Lyapunov stabilization inequalities associated with the possible state transition are integrated into a single matrix, and then the relaxed stabilization conditions are derived. The effectiveness of the proposed stabilization conditions are verified by an illustrative numerical example.

This study is organized as follows. Section 2 presents the considered system and the existing stabilization conditions. Section 3 develops the main result. Section 4 provides an illustrative example to demonstrate the utilization of the proposed method. Finally, Section 5 draws conclusions.

\section{Preliminary}

Consider a T-S fuzzy discrete system as follows:

$$
\begin{aligned}
& \text { Rule } i \text { : } \\
& \text { If } x_{1}(t) \text { is } M_{i 1}, x_{2}(t) \text { is } M_{i 2}, \ldots, x_{n}(t) \text { is } M_{i n} \text {, } \\
& \text { then } \boldsymbol{x}(t+1)=\boldsymbol{A}_{i} \boldsymbol{x}(t)+\boldsymbol{B}_{i} \boldsymbol{u}(t) \\
& \text { for } i=1,2, \ldots, r \text {. }
\end{aligned}
$$


where $\boldsymbol{A}_{i} \in \mathfrak{R}^{n \times n}$ and $\boldsymbol{B}_{i} \in \mathfrak{R}^{n \times m}$ are the system and input matrices, respectively. $\boldsymbol{x}(t) \in \mathfrak{R}^{n}$ and $\boldsymbol{u}(t) \in \mathfrak{R}^{m}$ are the state and input vectors, respectively. $M_{i j}$ is the fuzzy set. After defuzzification, the system is inferred as:

$$
\boldsymbol{x}(t+1)=\sum_{i=1}^{r} w_{i}(t)\left[\boldsymbol{A}_{i} \boldsymbol{x}(t)+\boldsymbol{B}_{i} \boldsymbol{u}(t)\right]
$$

where

$$
\begin{gathered}
w_{i}(t)=\frac{\prod_{j=1}^{n} M_{i j}\left(x_{j}(t)\right)}{\sum_{i=1}^{r} \prod_{j=1}^{n} M_{i j}\left(x_{j}(t)\right)} \\
0 \leq w_{i}(t) \leq 1 \\
\sum_{i=1}^{r} w_{i}(t)=1
\end{gathered}
$$

For a physical system, the control signal is constrained as follows:

$$
\|\boldsymbol{u}(t)\|_{2} \leq \mu
$$

The PDC control law [3] is usually employed to the system (2) as follows:

\section{Rule $i$ :}

If $x_{1}(t)$ is $M_{i 1}, x_{2}(t)$ is $M_{i 2}, \ldots, x_{n}(t)$ is $M_{i n}$, then $\boldsymbol{u}(t)=-\boldsymbol{F}_{i} \boldsymbol{x}(t)$

for $i=1,2, \ldots, r$.

After defuzzification, the controller is inferred as:

$$
\boldsymbol{u}(t)=-\sum_{i=1}^{r} w_{i}(t) \boldsymbol{F}_{i} \boldsymbol{x}(t)
$$

Apply the PDC control law to the system (2), then

$$
\boldsymbol{x}(t+1)=\sum_{i=1}^{r} \sum_{j=1}^{r} w_{i}(t) w_{j}(t)\left[\boldsymbol{A}_{i}-\boldsymbol{B}_{i} \boldsymbol{F}_{j}\right] \boldsymbol{x}(t)
$$

According the Lyapunov stability theorem with a common quadratic Lyapunov function $V(t)=\boldsymbol{x}^{T}(t) \boldsymbol{P} \boldsymbol{x}(t)$, the stabilization conditions for the T-S fuzzy discrete system (9) with constrained controls (6) are as follows:

Theorem 1[1, 4]: For $\|\boldsymbol{x}(0)\|_{2}<\phi$, the system (9) with constrained controls (6) is asymptotically stable if there are matrices $\boldsymbol{M}_{i}=\boldsymbol{F}_{i} \boldsymbol{P}^{-1}$ and $\boldsymbol{Q}=\boldsymbol{P}^{-1}>0$, such that the following inequalities are satisfied.

$$
\boldsymbol{Q}>\phi^{2} \boldsymbol{I}
$$

$$
\begin{gathered}
{\left[\begin{array}{cc}
\boldsymbol{Q} & * \\
\boldsymbol{M}_{i} & \mu^{2} \boldsymbol{I}
\end{array}\right]>0} \\
{\left[\begin{array}{cc}
\boldsymbol{Q} & * \\
\boldsymbol{A}_{i} \boldsymbol{Q}-\boldsymbol{B}_{i} \boldsymbol{M}_{i} & \boldsymbol{Q}
\end{array}\right]>0} \\
{\left[\begin{array}{c|c}
\frac{\boldsymbol{Q}}{\boldsymbol{A}_{i} \boldsymbol{Q}-\boldsymbol{B}_{i} \boldsymbol{M}_{j}+\boldsymbol{A}_{j} \boldsymbol{Q}-\boldsymbol{B}_{j} \boldsymbol{M}_{i}} & \boldsymbol{Q}
\end{array}\right]>0}
\end{gathered}
$$

where $i, j=1,2, \cdots, r ; i<j$. The asterisk denotes the transposed matrices for symmetric positions.

By using the following piecewise quadratic Lyapunov function, the relaxed stability conditions for the T-S fuzzy discrete system are expected.

$$
V(t)=\sum_{j=1}^{s} g_{j}(t) \boldsymbol{x}^{T}(t) \boldsymbol{P}_{j} \boldsymbol{x}(t)
$$

where

$$
g_{j}(t)= \begin{cases}1, & x(t) \in S_{j} \\ 0, & \text { otherwise }\end{cases}
$$

$\boldsymbol{S}_{j}$ denotes a subregion of the state space $\boldsymbol{X}$.

For a fuzzy system, the subregion division follows the firing of fuzzy rules, i.e., $0<M_{i j}\left(x_{j}\right) \leq 1$. Let the center of each fuzzy set be a dividing point in the state space $\boldsymbol{X}$, and then the subregions of a two-statevariable-system are illustrated as Fig. 1. Therefore, $\bigcup_{j=1}^{s} \boldsymbol{S}_{j}=\boldsymbol{X}$, and $\boldsymbol{S}_{i} \cap \boldsymbol{S}_{j}=\emptyset$ for $i \neq j$.

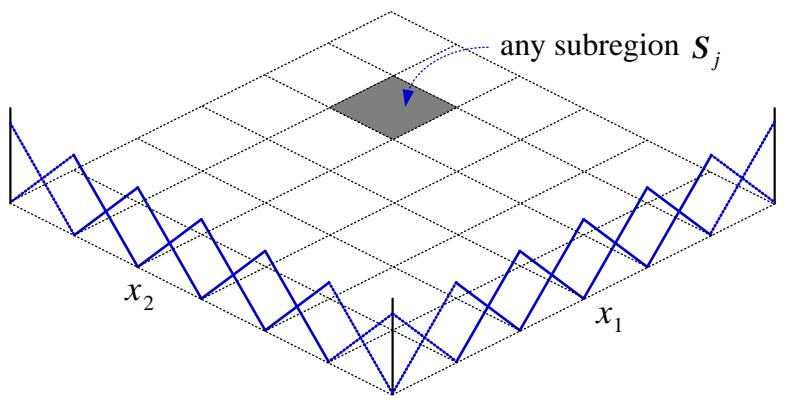

Fig. 1: Subregion division of the state space

Consequently, the T-S fuzzy discrete system is converted to an equivalent switching T-S fuzzy discrete system as follows:

\section{Region Rule $j$ :}

If $\boldsymbol{x}(t) \in S_{j}$, then

Local Fuzy Rule $\boldsymbol{L R}_{j l}$ :

$$
\begin{aligned}
& \text { If } x_{1}(t) \text { is } M_{j l 1}, x_{2}(t) \text { is } M_{j l 2}, \cdots, x_{n}(t) \text { is } M_{j l n}, \\
& \text { then } \boldsymbol{x}(t+1)=\boldsymbol{A}_{j l} \boldsymbol{x}(t)+\boldsymbol{B}_{j l} \boldsymbol{u}(t) \\
& \text { for } j=1,2, \cdots, s ; l=1,2, \cdots, \beta(j)
\end{aligned}
$$


After defuzzification, the switching T-S fuzzy discrete system is inferred as

$$
\boldsymbol{x}(t+1)=\sum_{j=1}^{s} \sum_{l=1}^{\beta(j)} g_{j}(t) w_{j l}(t)\left[\boldsymbol{A}_{j l} \boldsymbol{x}(t)+\boldsymbol{B}_{j l} \boldsymbol{u}(t)\right]
$$

where

$$
\begin{gathered}
w_{j l}(t)=\frac{\prod_{d=1}^{n} M_{j l d}\left(x_{d}(t)\right)}{\sum_{l=1}^{\beta(j)} \prod_{d=1}^{n} M_{j l d}\left(x_{d}(t)\right)} \\
0 \leq w_{j l}(t) \leq 1 \\
\sum_{l=1}^{\beta(j)} w_{j l}(t)=1
\end{gathered}
$$

Also, the PDC control law in the switching T-S fuzzy system form is considered.

\section{Region Rule $j$ :}

If $x(t) \in S_{j}$, then

\section{Local Fuzy Rule $\boldsymbol{L} \boldsymbol{R}_{j l}$ :}

$$
\begin{aligned}
& \text { If } x_{1}(t) \text { is } M_{j l 1}, x_{2}(t) \text { is } M_{j l 2}, \cdots, x_{n}(t) \text { is } M_{j l n} \text {, } \\
& \text { then } \boldsymbol{u}(t)=-\boldsymbol{F}_{j l} \boldsymbol{x}(t) \\
& \text { for } j=1,2, \cdots, s ; l=1,2, \cdots, \beta(j)
\end{aligned}
$$

After defuzzification, the controller is inferred as:

$$
\boldsymbol{u}(t)=-\sum_{j=1}^{s} \sum_{l=1}^{\beta(j)} g_{j}(t) w_{j l}(t) \boldsymbol{F}_{j l} \boldsymbol{x}(t)
$$

Apply the PDC control law to the system (17), then

$$
\begin{aligned}
& \boldsymbol{x}(t+1) \\
= & \sum_{j=1}^{s} \sum_{l=1}^{\beta(j)} \sum_{k=1}^{\beta(j)} g_{j}(t) w_{j l}(t) w_{j k}(t)\left[\boldsymbol{A}_{j l}-\boldsymbol{B}_{j l} \boldsymbol{F}_{j k}\right] \boldsymbol{x}(t) \\
= & \sum_{j=1}^{s} \sum_{l=1}^{\beta(j)} g_{j}(t) w_{j l}^{2}(t) \boldsymbol{G}_{j l} \boldsymbol{x}(t) \\
& +2 \sum_{j=1}^{s} \sum_{l=1}^{\beta(j)} \sum_{k>l}^{\beta(j)} g_{j}(t) w_{j l}(t) w_{j k}(t) \boldsymbol{\Lambda}_{j l k} \boldsymbol{x}(t)
\end{aligned}
$$

where $\boldsymbol{G}_{j l k}=\boldsymbol{A}_{j l}-\boldsymbol{B}_{j l} \boldsymbol{F}_{j k}$, and $\boldsymbol{\Lambda}_{j l k}=\left(\boldsymbol{G}_{j l k}+\boldsymbol{G}_{j k l}\right) / 2$.

According the Lyapunov stability theorem with the piecewise quadratic Lyapunov function (14), the stabilization conditions for the $\mathrm{T}-\mathrm{S}$ fuzzy discrete system (17) with constrained controls (6) are as follows:

Theorem 2[8]: For $\|\boldsymbol{x}(0)\|_{2}<\phi$, the system (17) with the constrained control (6) is asymptotically stable if there are matrices $\boldsymbol{M}_{j k}=\boldsymbol{F}_{j k} \boldsymbol{P}_{j}^{-1}$, and $\boldsymbol{Q}_{j}=\boldsymbol{P}_{j}^{-1}>0$, such that the following inequalities are satisfied.

$$
\begin{gathered}
\boldsymbol{Q}_{j}>\phi^{2} \boldsymbol{I} \\
{\left[\begin{array}{cc}
\boldsymbol{Q}_{j} & * \\
\boldsymbol{M}_{j l} & \mu^{2} \boldsymbol{I}
\end{array}\right]>0} \\
{\left[\begin{array}{cc}
\boldsymbol{Q}_{j} & * \\
\boldsymbol{A}_{j l} \boldsymbol{Q}_{j}-\boldsymbol{B}_{j l} \boldsymbol{M}_{j l} & \boldsymbol{Q}_{i}
\end{array}\right]>0} \\
{\left[\begin{array}{c|c}
\boldsymbol{Q}_{j} \\
\hline \boldsymbol{A}_{j l} \boldsymbol{Q}_{j}-\boldsymbol{B}_{j l} \boldsymbol{M}_{j k}+\boldsymbol{A}_{j k} \boldsymbol{Q}_{j}-\boldsymbol{B}_{j k} \boldsymbol{M}_{j l} \\
2
\end{array}\right.}
\end{gathered}
$$

where $i, j=1,2, \cdots, s ; l, k=1,2, \cdots, \beta(j) ; l<k$. The asterisk denotes the transposed matrices for symmetric positions.

It should be noted that the matrices $\boldsymbol{Q}_{j}=\boldsymbol{P}_{j}^{-1}$ and $\boldsymbol{Q}_{i}=\boldsymbol{P}_{i}^{-1}$ in Theorem 2 are with respect to the piecewise Lyapunov functions for $\boldsymbol{x}(t) \in \boldsymbol{S}_{j}$ and $\boldsymbol{x}(t+1) \in \boldsymbol{S}_{i}$, respectively. In a discrete system, the successive two states $\boldsymbol{x}(t)$ and $\boldsymbol{x}(t+1)$ may be in any subregions, consequently, $i, j=1,2, \cdots, s$ should be considered in Theorem 2 . If the relative positions of two consecutive states, $\boldsymbol{x}(t)$ and $\boldsymbol{x}(t+1)$, can be estimated, fewer $<j, i>$ pairs for Theorem 2 are expected. In next section, the stabilization conditions are reduced by estimating the maximum distance between two successive states.

\section{Main result}

For a physical system, the control signal is always constrained, as shown in (6). Therefore, from (17), if $\boldsymbol{x}(t) \in S_{j}$, the next state $\boldsymbol{x}(t+1)$ is bounded. In the subregion $S_{j}$, a state $x(t)$ can be represented as the convex combination of centers of the fuzzy sets $\boldsymbol{x}_{j c}^{v}$, as illustrated in Fig. 2.

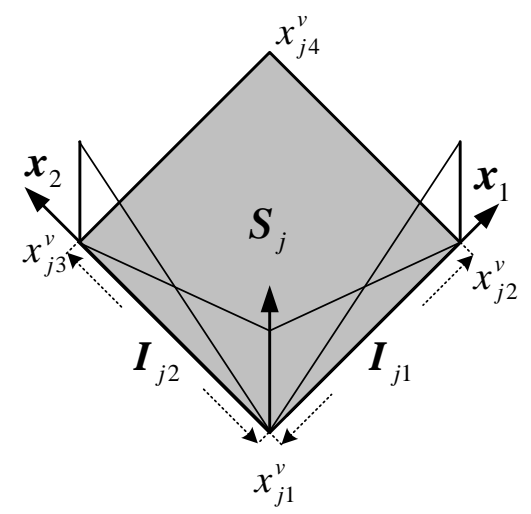

Fig. 2: Convex combination of centers of the fuzzy sets

$$
\boldsymbol{x}(t)=\sum_{c=1}^{2^{n}} w_{j c}(t) \boldsymbol{x}_{j c}^{v}, \text { for } \boldsymbol{x}(t) \in \boldsymbol{S}_{j}
$$


where

$$
\begin{gathered}
0 \leq w_{j c}(t) \leq 1 \\
\sum_{c=1}^{2^{n}} w_{j c}(t)=1
\end{gathered}
$$

According to (17), (28) and the constrained control (6), then

$$
\begin{aligned}
& \|\Delta \boldsymbol{x}(t)\|_{\infty} \\
& =\|\boldsymbol{x}(t+1)-\boldsymbol{x}(t)\|_{\infty} \\
& =\left\|\sum_{l=1}^{\beta(j)} w_{j l}(t)\left[\boldsymbol{A}_{j l} \boldsymbol{x}(t)+\boldsymbol{B}_{j l} \boldsymbol{u}(t)\right]-\boldsymbol{x}(t)\right\|_{\infty} \\
& \leq\left\|\sum_{l=1}^{\beta(j)} \sum_{c=1}^{2^{n}} w_{j l}(t) w_{j c}(t)\left[\boldsymbol{A}_{j l}-\boldsymbol{I}\right] \boldsymbol{x}_{j c}^{v}\right\|_{\infty} \\
& +\left\|\sum_{l=1}^{\beta(j)} w_{j l}(t) \boldsymbol{B}_{j l}\right\|_{\infty} \cdot \boldsymbol{\mu}, \quad \text { for } \boldsymbol{x}(t) \in \boldsymbol{S}_{j} .
\end{aligned}
$$

From (19)-(20) and (29)-(30),

$$
\begin{aligned}
& \|\Delta \boldsymbol{x}(t)\|_{\infty} \\
& \leq \max _{l, c}\left\|\left(\boldsymbol{A}_{j l}-\boldsymbol{I}\right) \boldsymbol{x}_{j c}^{v}\right\|_{\infty}+\max _{l}\left\|\boldsymbol{B}_{j l}\right\|_{\infty} \cdot \mu \\
& \leq \delta_{j} \cdot \min _{i}\left(\boldsymbol{I}_{j i}\right), \text { for } \boldsymbol{x}(t) \in \boldsymbol{S}_{j}
\end{aligned}
$$

where $\boldsymbol{I}_{j i}$ is the width of subregion $\boldsymbol{S}_{j}$, as shown in Fig. 2. $\delta_{j}$ is the minimum positive integer satisfying (32). Hence,

$$
\begin{aligned}
& \boldsymbol{x}(t+1) \in \boldsymbol{S}_{i}, \\
& \text { for } i=j \pm\left(1,2, \cdots, \delta_{j}\right), 0<i \leq s
\end{aligned}
$$

Define a set $\Omega$ to denote the possible state transition from $S_{j}$ to $S_{i}$.

$$
\begin{aligned}
\Omega= & \left\{<j, i>\mid x(t) \in \boldsymbol{S}_{j}, \boldsymbol{x}(t+1) \in \boldsymbol{S}_{i} ;\right. \\
& \left.j=1,2, \cdots, s ; i=j \pm\left(1,2, \cdots, \delta_{j}\right) ; i>0\right\}
\end{aligned}
$$

Apply the $\Omega$ in (34) to Theorem 2, the relaxed stabilization conditions for the T-S fuzzy discrete system with the constraint controls (6) is derived. In addition, the interactions among all fuzzy subsystems are integrated into a single matrix to relax the stabilization conditions.

Theorem 3: For $\|\boldsymbol{x}(0)\|_{2}<\phi$, the system (17) with the constrained controls (6) is asymptotically stable if there are matrices $\boldsymbol{M}_{j k}=\boldsymbol{F}_{j k} \boldsymbol{P}_{j}^{-1}, \boldsymbol{Y}_{j l k}=\boldsymbol{Y}_{j k l}^{T}, \boldsymbol{Y}_{j l}=\boldsymbol{Y}_{j l}^{T}$ and $\boldsymbol{Q}_{j}=\boldsymbol{P}_{j}^{-1}>0$, such that the following inequalities are satisfied.

$$
\boldsymbol{Q}_{j}>\phi^{2} \boldsymbol{I}
$$

$$
\begin{gathered}
{\left[\begin{array}{cc}
\boldsymbol{Q}_{j} & * \\
\boldsymbol{M}_{j l} & \mu^{2} \boldsymbol{I}
\end{array}\right]>0} \\
{\left[\begin{array}{cc}
\boldsymbol{Q}_{j}-\boldsymbol{Y}_{j l} & * \\
\boldsymbol{A}_{j l} \boldsymbol{Q}_{j}-\boldsymbol{B}_{j l} \boldsymbol{M}_{j l} & \boldsymbol{Q}_{i}
\end{array}\right]>0}
\end{gathered}
$$

$$
\begin{aligned}
& {\left[\begin{array}{c|c}
\boldsymbol{Q}_{j}-\boldsymbol{Y}_{j l k}-\boldsymbol{Y}_{j k l} & * \\
\hline \boldsymbol{A}_{j l} \boldsymbol{Q}_{j}-\boldsymbol{B}_{j l} \boldsymbol{M}_{j k}+\boldsymbol{A}_{j k} \boldsymbol{Q}_{j}-\boldsymbol{B}_{j k} \boldsymbol{M}_{j l} \\
2 & \boldsymbol{Q}_{i}
\end{array}\right]>0} \\
& \hat{\boldsymbol{Y}}_{j} \equiv\left[\begin{array}{cccc}
2 \boldsymbol{Y}_{j 1} & \boldsymbol{Y}_{j 12} & \cdots & \boldsymbol{Y}_{j 1 \beta(j)} \\
\boldsymbol{Y}_{j 21} & 2 \boldsymbol{Y}_{j 2} & \cdots & \boldsymbol{Y}_{j 2 \beta(j)} \\
\vdots & \vdots & \ddots & \vdots \\
\boldsymbol{Y}_{j \beta(j) 1} & \boldsymbol{Y}_{j \beta(j) 2} & \cdots & 2 \boldsymbol{Y}_{j \beta(j)}
\end{array}\right]>0
\end{aligned}
$$

where

$$
<j, i>\in \Omega
$$

$l, k=1,2, \cdots, \beta(j) ; l<k$. The asterisk denotes the transposed matrices for symmetric positions.

Remark 1: In Theorems 1 and 2, the stabilization conditions for $i, j=1,2, \cdots, s$ should be satisfied. In Theorem 3, however, only the conditions for $\langle j, i>\in \Omega$ defined as (34) are considered. Hence, the proposed Theorem 3 is more relaxed than Theorems 1 and 2 . However, if $\delta_{j}$ in (32) is too large, it would lead to $\Omega=\{\langle j, i\rangle \mid i, j=1,2, \cdots, s\}$ and then the proposed stabilization conditions regress to the traditional forms.

The control design procedure of Theorem 3 is summarized as follows:

Step 1: Convert the T-S fuzzy system (2) into a switching T-S fuzzy discrete system (17).

Step 2: Determine the possible state transition $\Omega$ through (32)-(34).

Step 3: List all stability conditions (35)-(39) according to $\Omega$.

Step 4: Solve the stabilization conditions by the existing linear matrix inequality tool.

\section{Illustrative Example}

This section demonstrates the effectiveness of the proposed stability conditions induced by considering the possible state transition $\Omega$.

Example 1: Consider the following T-S fuzzy discrete system with the constrained control $\|\boldsymbol{u}(t)\|_{2}<\mu=1$.

Rule $i$ :

If $x_{1}(t)$ is $M_{i 1}$, and $x_{2}(t)$ is $M_{i 2}$,

then $\boldsymbol{x}(t+1)=\boldsymbol{A}_{i} \boldsymbol{x}(t)+\boldsymbol{B}_{i} \boldsymbol{u}(t)$

for $i=1,2, \cdots, 8$ 
where

$$
\begin{aligned}
& \boldsymbol{A}_{1}=\left[\begin{array}{cc}
a & 0.4 \\
0.5 & 0.8
\end{array}\right], \boldsymbol{A}_{2}=\left[\begin{array}{ll}
0.9 & 0.5 \\
0.5 & 0.1
\end{array}\right], \boldsymbol{A}_{3}=\left[\begin{array}{ll}
0.9 & 0.3 \\
0.1 & 0.7
\end{array}\right], \\
& \boldsymbol{A}_{4}=\left[\begin{array}{ll}
0.7 & 0.2 \\
0.4 & 0.9
\end{array}\right], \boldsymbol{A}_{5}=\left[\begin{array}{ll}
0.9 & 0.3 \\
0.5 & 0.2
\end{array}\right], \boldsymbol{A}_{6}=\left[\begin{array}{ll}
0.8 & 0.3 \\
0.2 & 0.8
\end{array}\right], \\
& \boldsymbol{A}_{7}=\left[\begin{array}{ll}
0.8 & 0.2 \\
0.3 & 0.9
\end{array}\right], \boldsymbol{A}_{8}=\left[\begin{array}{ll}
0.8 & 0.1 \\
0.4 & 0.8
\end{array}\right],
\end{aligned}
$$

and

$$
\boldsymbol{B}_{1}=\left[\begin{array}{l}
b \\
0
\end{array}\right], \boldsymbol{B}_{2}=\left[\begin{array}{l}
0.6 \\
0.1
\end{array}\right]=\boldsymbol{B}_{3}=\boldsymbol{B}_{4}=\cdots=\boldsymbol{B}_{8} .
$$

In addition, the fuzzy sets of this system are shown as Fig. 3.
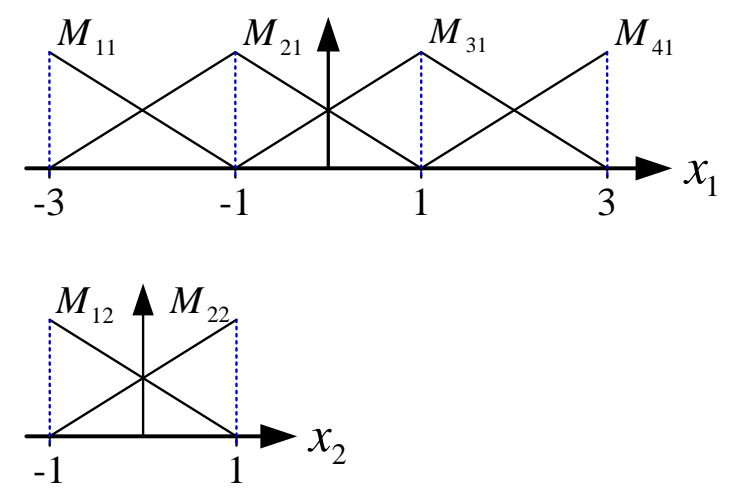

Fig. 3: Fuzzy sets of tis example

Then, the T-S fuzzy system is converted to an equivalent switching T-S fuzzy system:

\section{Region Rule $j$ :}

If $\boldsymbol{x}(t) \in \boldsymbol{S}_{j}$, then

$$
\begin{aligned}
& \text { Local Fuzy Rule } \boldsymbol{L} \boldsymbol{R}_{j l}: \\
& \text { If } x_{1}(t) \text { is } M_{j l 1}, x_{2}(t) \text { is } M_{j l 2}, \\
& \text { then } \boldsymbol{x}(t+1)=\boldsymbol{A}_{j l} \boldsymbol{x}(t)+\boldsymbol{B}_{j l} \boldsymbol{u}(t) \\
& j=1,2,3 ; l=1,2,3,4
\end{aligned}
$$

Accordingly, $\quad A_{11}=A_{1} \quad, \quad A_{12}=A_{21}=A_{2}$, $A_{22}=A_{31}=A_{3}, A_{32}=A_{4}, A_{13}=A_{5}, A_{14}=A_{23}=A_{6}$, $\boldsymbol{A}_{24}=\boldsymbol{A}_{33}=\boldsymbol{A}_{7}, \boldsymbol{A}_{34}=\boldsymbol{A}_{8}$, and $\boldsymbol{B}_{j l}=\left[\begin{array}{lll}0.6 & 0.1\end{array}\right]^{T}$, except $\boldsymbol{B}_{11}=\boldsymbol{B}_{1}$. As shown in Fig.3, $\boldsymbol{I}_{j i}=2$ and the centers of the fuzzy sets are

$$
\boldsymbol{x}_{11}^{v}=\left[\begin{array}{l}
-3 \\
-1
\end{array}\right], \boldsymbol{x}_{12}^{v}=\left[\begin{array}{l}
-1 \\
-1
\end{array}\right], \boldsymbol{x}_{13}^{v}=\left[\begin{array}{c}
-3 \\
1
\end{array}\right], \boldsymbol{x}_{14}^{v}=\left[\begin{array}{c}
-1 \\
1
\end{array}\right]
$$

$$
\begin{aligned}
& \boldsymbol{x}_{21}^{v}=\left[\begin{array}{l}
-1 \\
-1
\end{array}\right], \boldsymbol{x}_{22}^{v}=\left[\begin{array}{c}
1 \\
-1
\end{array}\right], \boldsymbol{x}_{23}^{v}=\left[\begin{array}{c}
-1 \\
1
\end{array}\right], \boldsymbol{x}_{24}^{v}=\left[\begin{array}{l}
1 \\
1
\end{array}\right] \\
& \boldsymbol{x}_{31}^{v}=\left[\begin{array}{c}
1 \\
-1
\end{array}\right], \boldsymbol{x}_{32}^{v}=\left[\begin{array}{c}
3 \\
-1
\end{array}\right], \boldsymbol{x}_{33}^{v}=\left[\begin{array}{l}
1 \\
1
\end{array}\right], \boldsymbol{x}_{34}^{v}=\left[\begin{array}{l}
3 \\
1
\end{array}\right]
\end{aligned}
$$

Hence, from (32), $\delta_{1}=2$ and $\delta_{2}=\delta_{3}=1$. Accordingly,

$$
\Omega=\left\{\begin{array}{l}
\langle 1,1\rangle,\langle 1,2\rangle,\langle 1,3\rangle,\langle 2,1\rangle, \\
\langle 2,2\rangle,\langle 2,3\rangle,\langle 3,2\rangle,\langle 3,2\rangle
\end{array}\right\}
$$

According to (43) and $\|x(0)\|_{2} \leq \phi=\sqrt{10}$, the stabilization conditions in Theorem 3 are derived and solved by the Matlab LMI toolbox [9]. Fig. 4 displays the feasible (a, b) pairs for the system (41) and the comparison with other literature [8]. Obviously, the proposed possible state transition set $\Omega$ and integrating matrix $\hat{\boldsymbol{Y}}_{j}$ bring the relaxed stabilization conditions for the T-S fuzzy system with the constrained control. Fig. 5 is the simulation result for the system (41) with $\mathrm{a}=0.6$ and $\boldsymbol{B}_{1}=\boldsymbol{B}_{2}$. The simulation shows that the system can be stabilized under the input constraint $\|\boldsymbol{u}(t)\|_{2}<\mu=1$.

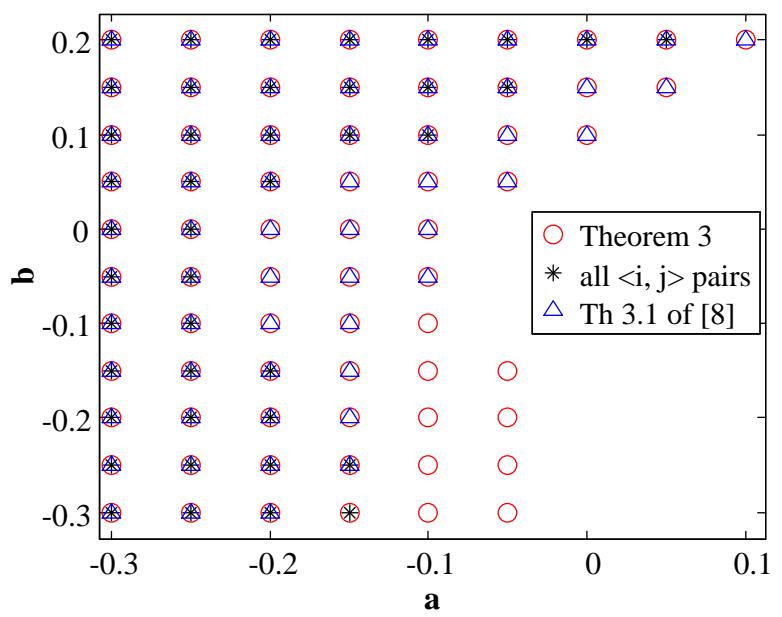

Fig. 4: The feasible $(a, b)$ pairs for this example.

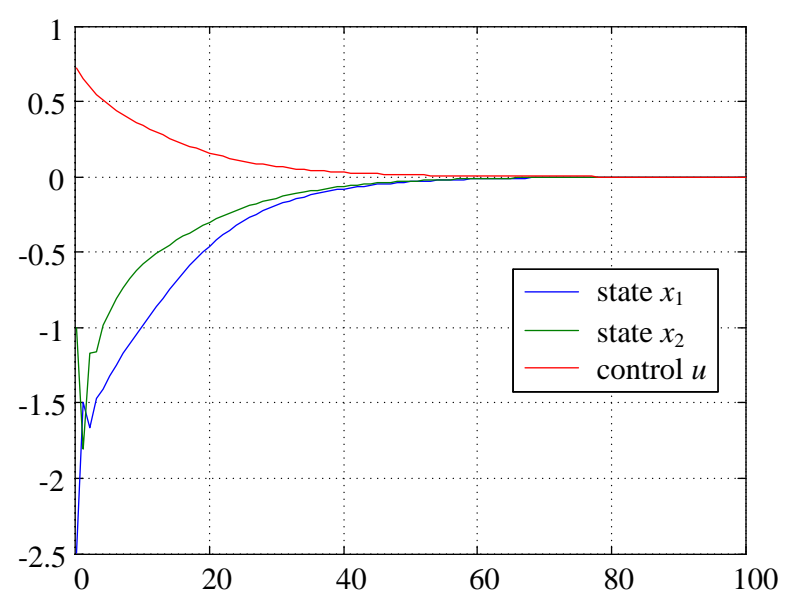

Fig. 5: Simulation result. 


\section{Conclusion}

In this paper, the stabilization conditions for the T-S fuzzy discrete system with the constrained control have been investigated. The piecewise Lyapunov function is adopted to derive the stabilization conditions. The possible state transition is considered to reduce the stabilization conditions. The correspond Lyapunov inequalities of the interactions among the state transition are integrated into a large slack matrix. An illustrated numerical example displays the effectiveness of the proposed method.

\section{Acknowledgment}

The authors like to thank Ministry of Science and Technology of Taiwan for its support under Contracts MOST 102-2221-E-008-085-MY3 and MOST 1032221-E-008-001-

\section{References}

[1] K. Tanaka and H. O. Wang. Fuzzy control systems design and analysis: a linear matrix inequality approach', John Wiley \& Sons, New York, 2001.

[2] G. Feng, A survey on analysis and design of modelbased fuzzy control systems, IEEE Trans. Fuzzy Systems, 14: 676--697, 2006.

[3] H. O. Wang, K. Tanaka, M. F. Griffin, An approach to fuzzy control of nonlinear systems: Stability and design issues, IEEE Trans. Fuzzy Systems, 4:14--23, 1996.

[4] K. Tanaka, M. Iwasaki, and H. O. Wang, Switching control of an R/C hovercraft: stabilization and smooth switching, IEEE Trans. Systems, Man, and Cybernetics, Part B, 31:853--863, 2001.

[5] K. Tanaka, H. Ohtake, and H. O. Wang, A practical design approach to stabilization of a 3-DOF RC helicopter, IEEE Trans. Control Systems Technology, 12:315--325, 2004.

[6] Y. J. Chen, W. J. Wang, and C. L. Chang, Guaranteed cost control for an overhead crane with practical constraints: Fuzzy descriptor system approach, Engineering Applications of Artificial Intelligence, 22:639--645, 2009.

[7] M. Tanaka, K. Yamaguchi, D. Ogura, Y. J. Chen and K. Tanaka, Nonlinear control of F16 aircraft via multiple nonlinear model generation for any trimmed equilibriums, International Journal of Fuzzy Systems, 16:140--152, 2014.

[8] C. H. Sun, S. W. Lin, and Y. T. Wang, Relaxed stabilization conditions for switching T-S fuzzy systems with practical constraints', International Journal of Innovative Computing, Information and Control, 8: 4133--4145, 2012.

[9] P. Gahinet, A. Nemirovski, A. Laub, and M. Chilali, LMI control toolbox, The Math Works Inc., 1994. 